Nonlin. Processes Geophys., 16, 189-196, 2009

www.nonlin-processes-geophys.net/16/189/2009/

(C) Author(s) 2009. This work is distributed under

the Creative Commons Attribution 3.0 License.

\title{
Spectral properties of electromagnetic turbulence in plasmas
}

\author{
D. Shaikh ${ }^{1}$ and P. K. Shukla ${ }^{2,3}$ \\ ${ }^{1}$ Department of Physics and Center for Space Plasma and Aeronomy Research (CSPAR), The University \\ of Alabama in Huntsville, Huntsville, AL-35899, USA \\ ${ }^{2}$ Institut für Theoretische Physik IV, Fakultät für Physik und Astronomie, Ruhr-Universität Bochum, \\ 44780 Bochum, Germany \\ ${ }^{3}$ SUPA Department of Physics, The University of Strathclyde, Glasgow, Scotland, UK
}

Received: 17 December 2008 - Revised: 5 March 2009 - Accepted: 5 March 2009 - Published: 12 March 2009

\begin{abstract}
We report on the nonlinear turbulent processes associated with electromagnetic waves in plasmas. We focus on low-frequency (in comparison with the electron gyrofrequency) nonlinearly interacting electron whistlers and nonlinearly interacting Hall-magnetohydrodynamic (H-MHD) fluctuations in a magnetized plasma. Nonlinear whistler mode turbulence study in a magnetized plasma involves incompressible electrons and immobile ions. Two-dimensional turbulent interactions and subsequent energy cascades are critically influenced by the electron whisters that behave distinctly for scales smaller and larger than the electron skin depth. It is found that in whistler mode turbulence there results a dual cascade primarily due to the forward spectral migration of energy that coexists with a backward spectral transfer of mean squared magnetic potential. Finally, inclusion of the ion dynamics, resulting from a two fluid description of the H-MHD plasma, leads to several interesting results that are typically observed in the solar wind plasma. Particularly in the solar wind, the high-timeresolution databases identify a spectral break at the end of the MHD inertial range spectrum that corresponds to a highfrequency regime. In the latter, turbulent cascades cannot be explained by the usual MHD model and a finite frequency effect (in comparison with the ion gyrofrequency) arising from the ion inertia is essentially included to discern the dynamics of the smaller length scales (in comparison with the ion skin depth). This leads to a nonlinear H-MHD model, which is presented in this paper. With the help of our 3-D H-MHD code, we find that the characteristic turbulent interactions in the high-frequency regime evolve typically on
\end{abstract}

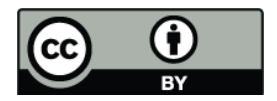

Correspondence to: D. Shaikh (dastgeer.shaikh@uah.edu)
kinetic-Alfvén time-scales. The turbulent fluctuation associated with kinetic-Alfvén interactions are compressive and anisotropic and possess equipartition of the kinetic and magnetic energies.

\section{Introduction}

Many laboratory and space plasmas contain multi-scale electromagnetic fluctuations. The latter include the electron whistlers and H-MHD fluctuations in a uniform magnetoplasma. While the wavelengths of the electron whistlers could be comparable with the electron skin depth, the HMHD fluctuations could have differential wavelengths when compared with the ion skin depth and the ion-sound gyroradius. In whistler mode turbulence, the electrons are incompressible and the ions form the neutralizing background in the plasma. On the other hand, in the H-MHD turbulence the compressibility of the electrons and ions cannot be ignored.

In uniform magnetized plasmas, one can have different types of electromagnetic waves. The latter include the low-frequency (in comparison with the electron gyrofrequency) electron whistlers as well as linearly coupled fast and slow magnetosonic and kinetic-Alfvén waves. The electron whistlers are described by the electron magnetohydrodynamics (EMHD) equations (Kingsep et al., 1990), and the role of whistlers in the EMHD turbulence is an unresolved issue (Shaikh et al., 2000a,b; Shaikh, 2004, 2008, 2009; Shaikh and Zank, 2003, 2005). Notably, EMHD in two dimensions exhibits dual cascade phenomena (Biskamp et al., 1996) similar to those of 2-D Navier-Stokes (Kraichnan, 1965; Frisch, 1995) and MHD dynamics (Fyfe and Montgomery, 1977; Biskamp and Bremer, 1994). However, because of

Published by Copernicus Publications on behalf of the European Geosciences Union and the American Geophysical Union. 
the complexity of nonlinear interactions in EMHD turbulence, mode coupling interactions are relatively more complex to comprehend. Like 2-D Navier-Stokes (Kraichnan, 1965; Frisch, 1995) and MHD (Fyfe and Montgomery, 1977; Biskamp and Bremer, 1994) systems, 2-D EMHD also exhibits dual cascade processes (Biskamp et al., 1996). The dual cascade phenomena, i.e. both the forward and inverse cascades, is however difficult to resolve numerically in a single energy spectrum because of a lack of spectral resolution in the inertial ranges. Therefore there exists considerably less numerical work on this issue as compared to various two-dimensional (decaying) turbulence systems. Numerical simulations of inverse cascade phenomenon, within the context of magnetohydrodynamics (MHD) turbulence, have previously been performed at a modest resolution of up to $1024^{2}$ Fourier modes (Biskamp and Bremer, 1994).

The dynamics of linearly coupled magnetic field-aligned Alfvén wave and obliquely propagating fast and slow magnetosonic waves and kinetic Alfvén waves are governed by the H-MHD equations. The solar wind plasma wave spectra corresponding to whistlers or high-frequency (in comparison with the ion gyrofrequency) kinetic-Alfvén wave possess a spectral break, the origin of which is not yet fully understood (Denskat et al., 1983; Goldstein et al., 1994; Leamon et al., 1998; Goldstein et al., 1996; Bale et al., 2005; Servidio et al., 2008). There are, however, some preliminary results available in the literature Shaikh and Shukla $(2008,2009)$.

In this paper, we present a survey of the spectral properties of the low-frequency multi-scale electromagnetic turbulence in plasmas. In Sect. 2, we begin by describing the whistler wave model based on two dimensional electron magnetohydrodynamic equations. Linear properties of whistler waves are described in this section. Section 3 contains nonlinear simulation results describing the wave spectra in small and large scale (in comparison with the electron skin depth) regimes. Section 4 describes the Hall- MHD counter part. The summary and conclusions are contained in Sect. 5.

\section{The whistler mode turbulence}

In an incompressible electron-MHD (E-MHD) plasma, collective oscillation of electrons exhibit electron whistlers. The electron Whistlers are widely observed in many space and laboratory plasmas. For instance, they are believed to be generated in the Earth's ionospheric region by lightning discharges and proceed in the direction of Earth's dipole magnetic field (Helliwell, 1965). They have also been recently detected in the Earth's radiation belt by the STEREO S/WAVES instrument (Cattell et al., 2008). Moreover, there are observations of Venus' ionosphere that reveal strong, circularly polarized, electromagnetic waves with frequencies near $100 \mathrm{~Hz}$. The waves appear as bursts of radiation lasting 0.25 to $0.5 \mathrm{~s}$, and have the expected properties of whistlermode signals generated by lightning discharges in Venus' clouds (Russell et al., 2007). These waves are also reported near the Earth's magnetopause (Stenberg et al., 2007) and the Cluster spacecraft encountered them during the process of magnetic reconnection in the Earth's magnetotail region (Wei et al., 2007). Upstream of collisionless shock, whistler waves are found to play a crucial role in heating the plasma ions (Scholer and Burgess, 2007). Their excitation and propagation are not only limited to the Earth's nearby ionosphere, but they are also found to be excited near the ionosphere of other planets such as in the radiation belts of Jupiter and Saturn (Bespalov, 2006). Whistlers are believed to be a promising candidate in transporting fields and currents in plasma opening switch (POS) devices (Mason et al., 1993), which operate on fast electron time scales. Similarly, electron whistlers have been known to drive the phenomenon of magnetic field line reconnection (Bulanov et al., 1992) in astrophysical plasmas (Zhou et al., 1996). Whistlers have also been investigated in several laboratory experiments (Stenzel, 1997, 1975; Stenzel and Urrutia, 1990; Stenzel et al., 1993), where they have been found to exhibit a variety of interesting features, such as an anisotropic propagation of the phase front, strong dispersion characteristics, interaction with plasma particles, etc. A few experimental features have also been confirmed by recent three-dimensional simulations (Eliasson and Shukla, 2007), where it has been reported that the polarity and the amplitude of the toroidal magnetic field, in agreement with the laboratory experiments, determine the propagation direction and speed of the whistlers. These are only a few examples amongst a large body of work devoted to the study of the whistlers. Despite the large amount of effort gone into understanding the existence and the propagation of whistlers, their linear dynamics is still debated, specially in the context of complex nonlinear processes. For example, the role of whistlers in the high- frequency turbulence and anisotropic spectral cascades has been debated recently (Shaikh et al., 2000a,b; Shaikh and Zank, 2003, 2005; Shaikh, 2004). In the following, we present a summary of the spectra of the whistler turbulence.

The electron whistler mode dynamics is essentially governed by the EMHD equations. The EMHD phenomena typically occur on the electron time scale (Kingsep et al., 1990), while the ions do not participate in the whistler mode dynamics (Shukla, 1978). Thus, the basic frequency regimes involved are $\omega_{c i} \ll \omega \ll \omega_{c e}$ (where $\omega_{c i}, \omega_{c e}$ are, respectively, the ion and electron gyrofrequencies, and $\omega$ is the characteristic whistler frequency), and the length scales are $c / \omega_{p i}<\ell<c / \omega_{p e}$, where $\omega_{p i}, \omega_{p e}$ are the ion and electron plasma frequencies, respectively. In whistlers, electrons carry currents, since immobile ions merely provide a neutralizing background to a quasi-neutral EMHD plasma. The electron momentum equation is

$m_{e} n \frac{d}{d t} V_{e}=-e n E-\frac{n e}{c} V_{e} \times B-\nabla P-\mu m_{e} n V_{e}$, 


$$
\begin{aligned}
& E=-\nabla \phi-\frac{1}{c} \frac{\partial A}{\partial t}, \\
& \nabla \times B=\frac{4 \pi}{c} J+\frac{1}{c} \frac{\partial E}{\partial t}, \\
& \frac{\partial n}{\partial t}+\nabla \cdot\left(n V_{e}\right)=0,
\end{aligned}
$$

where

$$
\frac{d}{d t}=\frac{\partial}{\partial t}+V_{e} \cdot \nabla \text {. }
$$

The remaining equations are $B=\nabla \times A, J=-e n V_{e}, \nabla \cdot B=0$. Here $m_{e}, n, V_{e}$ are the electron mass, density and fluid velocity respectively. $E, B$ respectively represent electric and magnetic fields and $\phi, A$ are electrostatic and electromagnetic potentials. The remaining variables and constants are, the pressure $P$, the collisional dissipation $\mu$, the current due to electrons flow $J$, and the velocity of light $c$. The displacement current in Ampére's law Eq. (3) is ignored, and the density is considered as constant throughout the analysis. The electron continuity equation can, therefore, be represented by a divergence-less electron fluid velocity $\nabla \cdot V_{e}=0$. The electron fluid velocity can then be associated with the rotational magnetic field through

$V_{e}=-\frac{c}{4 \pi n e} \nabla \times B$

By taking the curl of Eq. (1) and, after slight rearrangement of the terms, we obtain a generalized electron momentum equation in the following form.

$$
\frac{\partial P}{\partial t}-V_{e} \times(\nabla \times P)+\nabla \xi=-\mu m_{e} V_{e}
$$

where

$P=m_{e} V_{e}-\frac{e A}{c}$ and $\xi=\frac{1}{2} m_{e} V_{e} \cdot V_{e}+\frac{P}{n}-e \phi$.

The curl of Eq. (5) leads to a three-dimensional equation of EMHD describing the evolution of the whistler wave magnetic field,

$$
\frac{\partial}{\partial t} \Omega_{B}+V_{e} \cdot \nabla \Omega_{B}-\Omega_{B} \cdot \nabla V_{e}=\mu d_{e}^{2} \nabla^{2} B .
$$

where $\Omega_{B}=B-d_{e}^{2} \nabla^{2} B, d_{e}=c / \omega_{p e}$, the electron skin depth, is an intrinsic length-scale in the EMHD plasma. The threedimensional EMHD equations can be transformed into two dimensions by regarding variation in the $\hat{z}$-direction as ignorable i.e. $\partial / \partial z=0$, and separating the total magnetic field $B$ into two scalar variables, such that $B=\hat{z} \times \nabla \psi+b \hat{z}$. Here $\psi$ and $b$ respectively present perpendicular and parallel components of the wave magnetic field. The corresponding equations of these components can be written in a normalized form as follows,

$$
\frac{\partial}{\partial t} \Omega_{\psi}+\hat{z} \times \nabla b \cdot \nabla \Omega_{\psi}-B_{0} \frac{\partial}{\partial y} b=0
$$

$\frac{\partial}{\partial t} \Omega_{B}-d_{e}^{2} \hat{z} \times \nabla b \cdot \nabla \nabla^{2} b+\hat{z} \times \nabla \psi \cdot \nabla \nabla^{2} \psi+B_{0} \frac{\partial}{\partial y} \nabla^{2} \psi=0$,

where $\Omega_{\psi}=\psi-d_{e}^{2} \nabla^{2} \psi, \Omega_{B}=b-d_{e}^{2} \nabla^{2} b$ The length and time scales are normalized respectively by $d_{e}$ and $\omega_{c e}$, whereas magnetic field is normalized by a typical mean $B_{0}$. The linearization of Eqs. (7) and (8) about a constant magnetic field $B_{0}$ yields the dispersion relation for the whistlers, the normal mode of oscillation in the EMHD frequency regime, and is given by

$\omega_{k}=\omega_{c_{0}} \frac{d_{e}^{2} k_{y} k}{1+d_{e}^{2} k^{2}}$,

where $\omega_{c_{0}}=e B_{0} / m c$ and $k^{2}=k_{x}^{2}+k_{y}^{2}$. From the set of the EMHD Eqs. (7) and (8), there exists an intrinsic length scale $d_{e}$, the electron inertial skin depth, which divides the entire spectrum into two regions; namely short scale $\left(k d_{e}>1\right)$ and long scale $\left(k d_{e}<1\right)$ regimes. In the regime $k d_{e}<1$, the linear frequency of whistlers is $\omega_{k} \sim k_{y} k$ and the waves are dispersive. Conversely, dispersion is weak in the other regime $k d_{e}>1$ since $\omega_{k} \sim k_{y} / k$ and hence the whistler wave packets interact more like the eddies of hydrodynamical fluids.

\section{Energy spectra in whistler turbulence}

By virtue of $d_{e}$, there exists two inertial ranges that correspond to smaller $k d_{e}>1$ and larger $k d_{e}<1$ length-scales in the EMHD turbulence. Correspondingly, forward cascade turbulent spectra in these regimes exhibit $k^{-5 / 3}$ and $k^{-7 / 3}$ respectively as shown in Fig. (1). The whistler spectrum $k^{-7 / 3}$ is produced essentially by fluctuations in electron fluid while ions are at rest. The observed spectra in our simulations depicted as in Fig. (1) can be explained on the basis of Kolmogorov phenomenology as follows.

The regime $k d_{e}>1$ in EMHD turbulence corresponds essentially to a hydrodynamic regime because the energy spectrum is dominated by the shorter length-scale turbulent eddies that give rise to a characteristic spectrum of an incompressible hydrodynamic fluid. The group velocity of whistlers, in this regime, is small and hence it is expected that the whistler effect cannot be present. For $k d_{e}>1$, the first term in the magnetic potential can be dropped and thus

$A \sim\left(\nabla^{2} \psi\right)^{2}$.

Similarly the dominating terms in the energy are

$E \sim(\nabla b)^{2}+\left(\nabla^{2} \psi\right)^{2}$.

This implies

$E \sim(\nabla b)^{2} \sim\left(\nabla^{2} \psi\right)^{2}$.

Thus for $k d_{e}>1, E \sim A$. In this case both magnetic potential and the energy cascades turn out to be identical leading to the same spectral index, i.e. $k^{-5 / 3}$, in both direct and inverse cascade regimes. This is shown in Fig. (1) (left panel). 

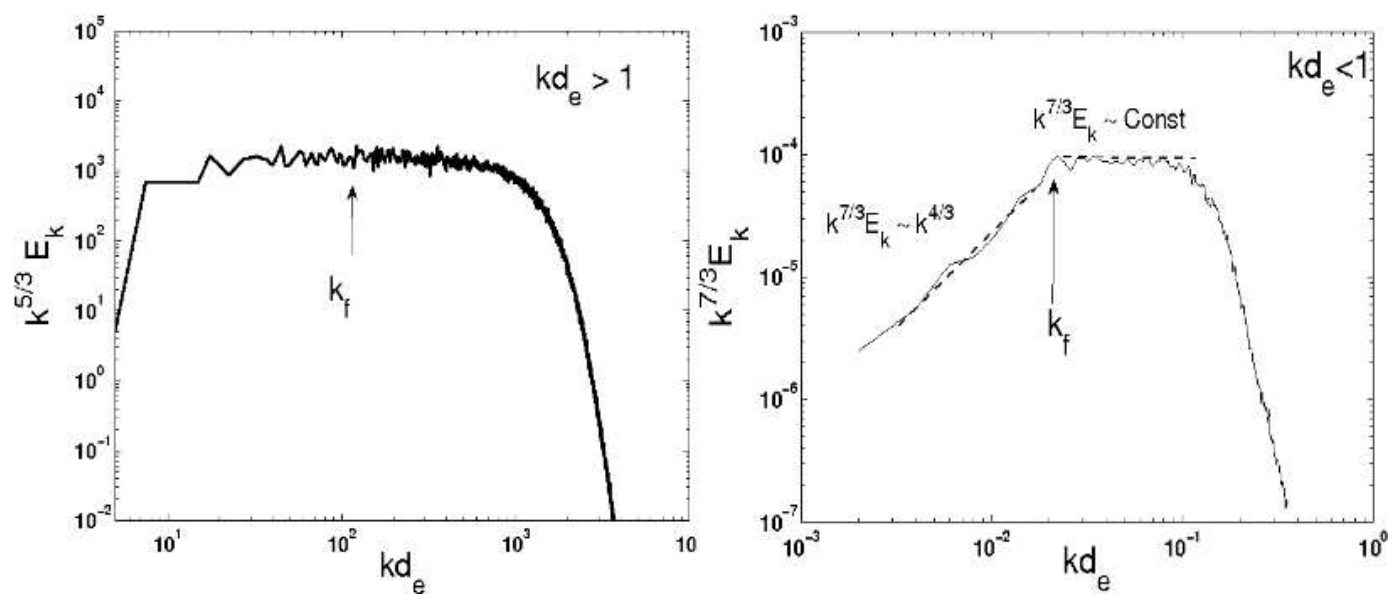

Fig. 1. (left) Driven 2-D whistler wave turbulence with the forcing band identified by the $k_{f}$ modes. The spectral resolution is $5120^{2}$. Notice that no spectral break exists across the forcing modes, which agrees with the Kolmogorov theory. Across the $k_{f}$ modes, the forward cascade of energy $\left(k>k_{f}\right)$ and the inverse cascade of mean magnetic potential $\left(k<k_{f}\right)$ yield identical spectral indices and are close to $E_{k} \sim k^{-5 / 3}$. We plot the compensated energy spectrum $k^{5 / 3} E_{k} \sim \varepsilon^{2 / 3} \sim$ Const to show inertial range modes along a straight line that is indicative of constant energy dissipation rates in steady state driven dissipative turbulence. (right) The inverse and forward cascade regimes are identified in 2 $\mathrm{D}$ driven whistler wave turbulence. The compensated energy spectrum in the inverse cascade regime $k^{7 / 3} E_{\mathrm{inv}} \sim \varepsilon^{2 / 3} k^{7 / 3} k^{-1} \sim \varepsilon^{2 / 3} k^{4 / 3}$, whereas the forward cascade is given by $k^{7 / 3} E_{f w d} \sim$ Const. The approximate error in the spectral slope is about \pm 0.072 . The spectral break, separating a forward and an inverse cascade regimes, corresponds to a hump above the forcing modes $k_{f}$ that results from a direct excitation of the Fourier modes in driven whistler wave turbulence.

In EMHD the eddy velocity $(v)$ in the $x-y$ plane is characterized by $\hat{z} \times \nabla b$. Thus the typical velocity with a scale size $\ell$ can be represented by $v \simeq b_{\ell} / \ell$. The eddy scrambling time is then given by

$\tau \sim \frac{\ell}{v} \sim \frac{\ell^{2}}{b_{\ell}}$.

In the limit of $k d_{e}<1$, the mean square magnetic potential is, $A \sim \psi^{2} \sim b_{\ell}^{2} \ell^{2}$.

The second similarity follows from the assumption of an equipartition of energy in the axial and poloidal components of magnetic field. This assumption has been invoked in some earlier works too, and would be exact if the EMHD turbulence comprised of randomly interacting whistler waves only. To determine the scaling behaviour of the spectrum in the magnetic potential cascade regime (i.e. for $k<k_{f}, k_{f}$ representing the forcing scales), the rate of $A$ transfer is given by the relation,

$\varepsilon=\frac{A}{\tau}=b_{\ell}^{3}$,

and the mean square magnetic potential per unit wave number is

$A_{k}=b_{\ell}^{2} \ell^{3}$.

The locality of the spectral cascade in the wavenumber space yields $A_{k}=\varepsilon^{\alpha} k^{\beta}$. On equating the powers of $b_{\ell}$ and $\ell$, we obtain $\alpha=2 / 3$ and $\beta=-3$. In $k d_{e}<1$ regime, the expression for the energy can be approximated as $E \sim b^{2} \sim(\nabla \psi)^{2}$. This further implies that $E=A / \ell^{2}$ and

$E_{k} \sim k^{-1}$.

A similar analysis in the other regime of $k>k_{f}$ where energy cascade is local in wave number space leads to an energy spectrum with the index of $-7 / 3$ such that

$E_{k} \sim k^{-7 / 3}$.

The compensated spectra of Fig. (1) (right panel) are consistent with above description.

In the following section, we shall include the ion dynamics that correspond to turbulence scales in which $k d_{i}>1$, where $d_{i}$ is ion skin depth. This regime of the magnetofluid dynamics is referred to as the Hall MHD, and it stems from the combined motion of the electron and ion fluids amidst predominant density fluctuations that evolves on Kinetic-Alfvén wave (KAW) time (and length) scales.

\section{The high-frequency kinetic-Alfvén inertial regime}

The high-frequency kinetic-Alfvén regime of the plasma results in the solar wind turbulence when plasma wave fluctuations comprise a multitude of time and space scales that are shorter than the ion gyroperiod $\left(2 \pi / \Omega_{c i}\right)$ and comparable (or longer) to (than) the ion thermal gyroradius/ion-sound gyroradius or shorter than the ion skin depth, respectively. These 
electromagnetic fluctuations cannot be described by the usual magnetohydrodynamic (MHD) model (Denskat et al., 1983; Goldstein et al., 1994; Leamon et al., 1998; Goldstein et al., 1996; Shaikh and Shukla, 2008, 2009) which involve the characteristic time scale much longer than the ion gyroperiod. Typically, reconnection of magnetic field lines near the solar corona (Bhattacharjee, 2004), Earth's magnetosphere and in many laboratory plasma devices (Bhattacharjee et al., 2001) are other avenues where collisionless or kinetic processes call for inclusion of the finite Larmor radius and finite frequency effects in the standard MHD model. In particular, in the context of the solar wind plasma, higher time resolution databases identify a spectral break near the end of the MHD inertial range spectrum that corresponds to a highfrequency $\left(>\Omega_{c i}\right)$ regime where turbulent cascades are not explainable by Alfvénic cascades. This refers to a secondary inertial range where turbulent cascades follow a $k^{-7 / 3}$ (where $k$ is a typical wavenumber) spectrum in which the characteristic fluctuations evolve typically on kinetic-Alfvén time scales (Howes et al., 2008; Matthaeus et al., 2008). The onset of the second or the kinetic-Alfvén inertial range still eludes our understanding of solar wind fluctuations (Leamon et al., 1998; Shaikh and Shukla, 2008, 2009). The mechanism leading to the spectral break has been thought to be either mediated by the kinetic- Alfvén waves (KAWs), or damping of ion-cyclotron waves, or dispersive processes, or other implicit nonlinear interactions associated with kinetic effects in solar wind plasmas (Denskat et al., 1983; Goldstein et al., 1994, 1996; Leamon et al., 1998; Shaikh and Shukla, 2008, 2009). Motivated by these issues, we have developed three dimensional, time dependent, compressible, non-adiabatic, driven and fully parallelized Hall-magnetohydrodynamic (HMHD) simulations (Shaikh and Shukla, 2008, 2009) to investigate turbulent spectral cascades in collisionless space plasmas.

In the high-frequency regime, $\omega>\Omega_{c i}$, the inertialess electrons contribute to the electric field which is dominated essentially by the Hall term corresponding to $J \times B$ force. The latter, upon substituting in the ion momentum equation, modifies the ion momentum, the magnetic field and total energy in a manner to introduce a high-frequency $\left(\omega>\Omega_{c i}\right)$ and small scale $\left(k_{\perp} \rho_{L}>1\right.$, where $\rho_{L}$ is ion thermal gyroradius) plasma motions. The characteristic length scales $\left(k^{-1}\right)$ associated with the plasma motions are smaller than the ion gyroradius $\left(\rho_{L}\right)$. The quasi-neutral solar wind plasma density $(\rho)$, velocity $(U)$, magnetic field $(B)$ and total pressure $\left(P=P_{e}+P_{i}\right)$ fluctuations can then be cast into a set of the Hall-MHD equations, given by

$$
\begin{aligned}
& \frac{\partial \rho}{\partial t}+\nabla \cdot(\rho U)=0, \\
& \rho\left(\frac{\partial}{\partial t}+U \cdot \nabla\right) U=-\nabla P+\frac{1}{c} J \times B \\
& \frac{\partial B}{\partial t}=\nabla \times\left(U \times B-d_{i} \frac{J \times B}{\rho}\right)+\eta \nabla^{2} B,
\end{aligned}
$$

$$
\frac{\partial e}{\partial t}+\nabla \cdot\left(\frac{1}{2} \rho U^{2} U+\frac{\gamma}{\gamma-1} \frac{P}{\rho} \rho U+\frac{c}{4 \pi} E \times B\right)=0
$$

where

$e=\frac{1}{2} \rho U^{2}+\frac{P}{(\gamma-1)}+\frac{B^{2}}{8 \pi}$

is the total energy of the plasma that contains both the electron and ion motions. All the dynamical variables are functions of three space and a time, i.e. $(x, y, z, t)$, co-ordinates. Equations (9) to (12) are normalized by typical length $\ell_{0}$ and time $t_{0}=\ell_{0} / v_{0}$ scales in our simulations, where $v_{0}=B_{0} /\left(4 \pi \rho_{0}\right)^{1 / 2}$ is Alfvén velocity such that $\bar{\nabla}=\ell_{0} \nabla, \partial / \partial \bar{t}=t_{0} \partial / \partial t, \bar{U}=U / v_{0}, \bar{B}=B / v_{0}\left(4 \pi \rho_{0}\right)^{1 / 2}$, $\bar{P}=P / \rho_{0} v_{0}^{2}, \bar{\rho}=\rho / \rho_{0}$. The parameters $\mu$ and $\eta$ correspond respectively to ion-electron viscous drag term and magnetic field diffusivity. While the viscous drag modifies the dissipation in plasma momentum in a nonlinear manner, the magnetic diffusion damps the small scale magnetic field fluctuations linearly. The magnetic field is measured in the unit of Alfvén velocity. The dimensionless parameter in magnetic field Eq. (11) i.e. ion skin depth $\bar{d}_{i}=d_{i} / \ell_{0}, d_{i}=C / \omega_{p i}$ is associated with the Hall term. This means the ion inertial scale length $\left(d_{i}\right)$ is a natural or an intrinsic length scale present in the Hall MHD model which accounts for finite Larmour radius effects corresponding to high frequency oscillations in $k d_{i}>1$ regime. Clearly, the Hall force dominates the magnetoplasma dynamics when $1 / \rho(J \times B)>U \times B$ term in Eq. (11) which in turn introduces time scales corresponding to the high frequency plasma fluctuations in $k d_{i}>1$ regime. Furthermore, our model includes a full energy equation (Eq. 12) unlike an adiabatic relation between the pressure and density. The use of energy equation enables us to study a self-consistent evolution of turbulent heating resulting from nonlinear energy cascades in the solar wind plasma.

In the linear regime, the Hall-MHD equations (without the energy equation) admit a dispersion relation,

$\left(\omega^{2}-k_{z}^{2} V_{A}^{2}\right) D_{m}(\omega, k)=\omega^{2}\left(\omega^{2}-k^{2} V_{s}^{2}\right) k_{z}^{2} k^{2} V_{A}^{4} / \omega_{c i}^{2}$,

which exhibits coupling among the fast and slow magnetosonic waves given by the solutions of

$D_{m}(\omega, k)=\omega^{4}-\omega^{2} k^{2}\left(V_{A}^{2}+V_{s}^{2}\right)+k_{z}^{2} k^{2} V_{A}^{2} V_{s}^{2}=0$,

where $\omega$ is the frequency, $k\left(=k_{\perp}+k_{z} \hat{z}\right.$ is the wave vector, the subscripts $\perp$ and $z$ represent the components across and along the external magnetic fields $B_{0} \hat{z}$, and $V_{A}$ and $V_{s}$ are the Alfvén and effective sound speeds, respectively. The warm HMHD plasma thus supports a great variety of waves (e.g. magnetic field-aligned non-dispersive Alfvén waves, obliquely propagating fast and slow magnetohydrodynamic waves, obliquely propagating dispersive kinetic Alfvén waves and whistlers) having different wavelengths (comparable to the ion skin depth associated with the Hall drift, the ion sound gyroradius associated with the electron 


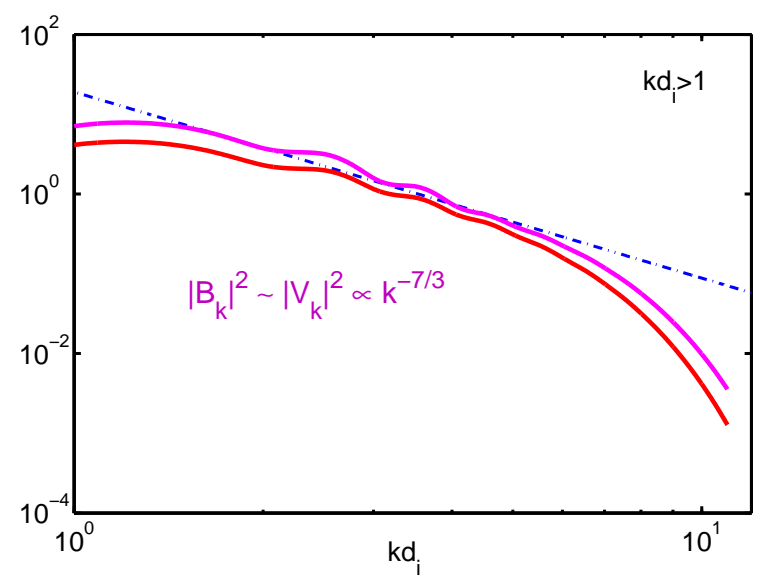

Fig. 2. Inertial range turbulent spectra of magnetic and velocity field fluctuations are shown. These fluctuations closely follow a $k^{-7 / 3}$ spectrum in kinetic Alfvén regime where $k d_{i}>1$. The simulation parameters are: spectral resolution is $128^{3}$, $\eta=\mu=10^{-3}, M_{A}=\beta=1.0, k d_{i} \geq 1, L_{x}=L_{y}=L_{z}=2 \pi$.

pressure and the perpendicular ion inertia/ion polarization drift). Interestingly, in the $\omega<\Omega_{c i}$ regime, which is predominantly known to be an Alfvénic regime, Howes et al. (2008) noted the possibility of occurance of highly obliquely propagating KAWs (with $\omega \ll \Omega_{c i}$ ) thereby questioning the role of damping of ion cyclotron waves in the onset of spectral breakpoint. While our simulations described in Fig. (2) may contain the KAWs corresponding to $\omega \ll \Omega_{c i}$, their specific contribution in the spectral cascades depicted in Fig. (2) and Fig. (3) is not identifiable. We therefore leave this study to a future investigation.

The nonlinear spectral cascades in the KAW regime lead to a secondary inertial range in the vicinity of modes $k d_{i} \simeq 1$ in that the solar wind magnetic, velocity and field fluctuations follow turbulent spectra close to $k^{-7 / 3}$. This is shown in Fig. (2). The characteristic turbulent spectra in the KAW regime is steeper than that of MHD inertial range which follows a $k^{-\alpha}$ where $\alpha$ is $5 / 3$ or $3 / 2$. The onset of the secondary inertial range is constantly debated because of the presence of multiple processes in the KAW regime that include, for instance, the dispersion, damping of ion cyclotron waves, turbulent dissipation or due to fast or slow magnetosonic perturbations and etc. In the context of our simulations, the observed $k^{-7 / 3}$ in the solar wind turbulent plasma can be understood from the energy cascades affected by the Hall forces. The latter are one of the potential candidates that may be responsible for a $k^{-7 / 3}$ spectrum in the KAW inertial range regime.

To understand the observed $k^{-7 / 3}$ spectrum in the KAW inertial range regime in solar wind plasma and in our simulations, we invoke Kolmogorov and Kraichnan like phenomenologies (Kolmogorov, 1941; Kraichnan, 1965) in Hall MHD plasma. The time scale associated with Hall MHD is $\tau_{\mathrm{H}}$ smaller than that of MHD $\tau_{\mathrm{MHD}}$. The nonlinear cas-

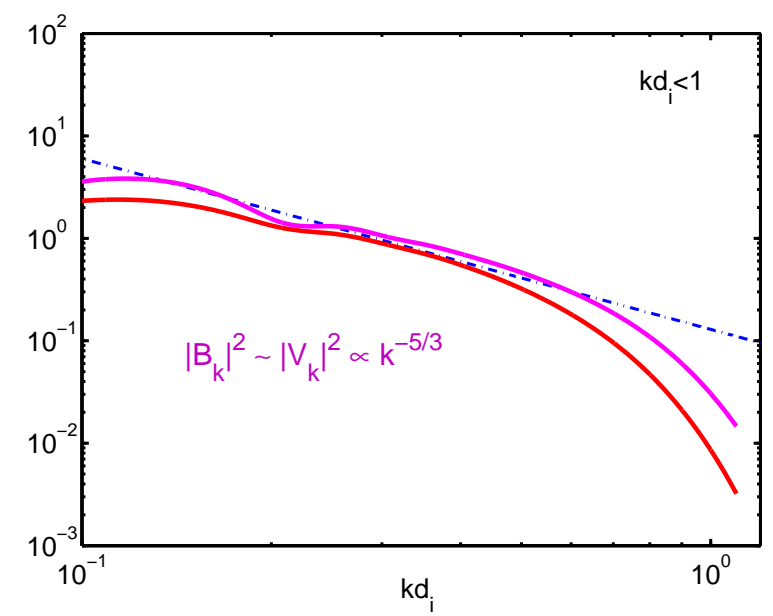

Fig. 3. Inertial range turbulent spectra in $k d_{i}<1$ regime. Shown are the magnetic and velocity field fluctuations along with that of the density fluctuation. These fluctuations closely follow a $k^{-5 / 3}$ omnidirectional spectrum in the Alfvénic regime. Simulation parameters are the same as in Fig. (2), except $d_{i}=0.05$.

cades in the MHD turbulence are governed typically by $\tau_{\mathrm{MHD}} \sim\left(k u_{k}\right)^{-1}$, where $u_{k}$ is the velocity field in the $k$ space. By contrast, the spectral transfer of the turbulent energy in the solar wind (Hall MHD) plasma is $\tau_{\mathrm{H}} \sim\left(k^{2} B_{k}\right)^{-1}$. The energy transfer rates in the KAW regime are therefore $\varepsilon \sim u_{k}^{2} / \tau_{\mathrm{H}}$. On substituting the turbulent equipartition relation between the the velocity and magnetic fields $B_{k}^{2} \sim u_{k}^{2}$, the energy transfer rates turn out to be $\varepsilon \sim k^{2} B_{k}^{3}$. The use of the turbulent equipartition is justified from the observations and it is also seen in our 3-D simulations (see Fig. 5). Applying the Kolmogorov's phenomenology (Kolmogorov, 1941; Kraichnan, 1965) that the energy cascades in the inertial range are local and that they depend on the Fourier modes and the energy dissipation rate, we obtain $k^{-1} B_{k}^{2} \sim\left(B_{k}^{3} k^{2}\right)^{\alpha} k^{\beta}$. Equating the indices of the common bases, we get $\alpha=2 / 3$ and $\beta=-7 / 3$. This results in an energy spectrum $E_{k} \propto k^{-7 / 3}$ that is consistent with our 3-D simulations (see Fig. 2) and the observations. On the other hand, the use of $\tau_{\mathrm{MHD}}$ in estimating the energy dissipation rates retrieves the inertial range MHD spectrum. This indicates that the Hall effects may be responsible for the spectral steepening in the solar wind plasma fluctuations in the $k d_{i}>1$ regime. It is worth noted that our simulations in $k d_{i}<1$ exhibit MHD like $k^{-5 / 3}$ omnidirectional Kolmogorov-like (Kolmogorov, 1941) spectrum as shown in Fig. (3).

\section{Conclusions}

In summary, we have presented a survey of the spectral properties of low-frequency electromagnetic turbulence in uniform magnetized plasmas. Specifically, we have focused on the electron whistlers, and high-frequency kinetic Alfvén 
waves. While for the electron whistlers the motion of ions is unimportant, the ion dynamics plays an essential role in the high-frequency KAW turbulence. We have developed codes to numerically solve the governing nonlinear equations for the whistler mode and KAW turbulences. Our simulations for the whistler mode turbulence depict a dual cascade process between the energy and mean magnetic potential in consistent with a Kolmogorov phenomenology. Furthermore, the high-frequency kinetic Alfvén regime, typically observed in the solar wind plasma, is studied by means of 3-D simulations. Our 3-D simulation results exhibit that the characteristic turbulent interactions in the high-frequency regime evolve typically on the Hall time-scales and modify the inertial range spectra that eventually leads to the observed spectral break in the solar wind.

Acknowledgement. The support of NASA(NNG-05GH38) and NSF (ATM-0317509) grants is acknowledged.

This work was partially supported by the Deutsche Forschungsgemeinschaft through the project $\mathrm{SH} 21 / 3-1$ of the Forschergruppe 1048.

Edited by: P.-L. Sulem

Reviewed by: M. Goldstein and another anonymous referee

\section{References}

Bale, S. D., Kellogg, P. J., Mozer, F. S., Horbury, T. S., and Reme, H.: Measurement of the Electric Fluctuation Spectrum of Magnetohydrodynamic Turbulence, Phys. Rev. Lett. 94, 215002, doi:10.1103/PhysRevLett.94.215002, 2005.

Bespalov, P. A.: Excitation of whistler waves in three spectral bands in the radiation belts of Jupiter and Saturn, European Planetary Science Congress, Berlin, Germany, 18-22 September 2006, p. 461, 2006.

Biskamp, D., Schwarz, E., and Drake, J. F.: Two-dimensional electron magnetohydrodynamic turbulence, Phy. Rev. Lett., 761264 1267, 1996.

Biskamp, D. and Bremer, U.: Dynamics and Statistics of Inverse Cascade Processes in 2-D Magnetohydrodynamic Turbulencem, Phys. Rev. Lett., 72, 3819-3822, 1994.

Bhattacharjee, A.: Impulsive Magnetic Reconnection in the Earth's Magnetotail and the Solar Corona, Annu. Rev. Astron. Astrophys, 42, 365-384, 2004.

Bhattacharjee, A., Ma, Z. W., and Wang, X.: Recent developments in collisionless reconnection theory: Applications to laboratory and space plasmas, Phys. Plasmas, 8, 1829-1839, 2001.

Bulanov, S. V., Pegoraro, F., and Sakharov, A. S.: Magnetic reconnection in electron magnetohydrodynamics, Phys. Fluids, B., 4 2499-2508, 1992.

Cattell, C., Wygant, J. R., Goetz, K., Kersten, K., Kellogg, P. J., von Rosenvinge, T., Bale, S. D., Roth, I., Temerin, M., Hudson, M. K., Mewaldt, R. A., Wiedenbeck, M., Maksimovic, M., Ergun, R., Acuna, M., and Russell, C. T.: Discovery of very large amplitude whistler-mode waves in Earth's radiation belts, Geophys. Res. Lett., 35, L01105, doi:10.1029/2007GL032009, 2008.

Denskat, K. U., Beinroth, H. J., and Neubauer, F. M.: Interplanetary magnetic field power spectra with frequencies from $2.4 \times 10$ to the -5 th HZ to $470 \mathrm{HZ}$ from HELIOS-observations during solar minimum conditions, J. Geophys. Res., 54, 60-67, 1983.

Eliasson, B. and Shukla, P. K.: Dynamics of Whistler Spheromaks in Magnetized Plasmas, Phys. Rev. Lett., 99, 205005, doi:10.1103/PhysRevLett.99.205005, 2007.

Frisch, U.: Turbulence: The Legacy of A. N. Kolmogorov (Cambridge University Press, Cambridge, 1995), 1995.

Fyfe, D., Montgomery, D., and Joyce, G.: Dissipative, forced turbulence in two-dimensional magnetohydrodynamics, J. Plasma Phys. 17, 369-398, 1977.

Goldstein, M. L., Roberts, D. A., and Fitch, C. A.: Properties of the fluctuating magnetic helicity in the inertial and dissipation ranges of solar wind turbulence, J. Geophys. Res., 99, 1151911538, 1994.

Ghosh, S., Siregar, E., Roberts, D. A., and Goldstein, M. L.: Simulation of high-frequency solar wind power spectra using Hall magnetohydrodynamics, J. Geophys. Res., 101, 2493-2504, 1996.

Howes, G. G., Dorland, W., Cowley, S. C., Hammett, G. W., Quataert, E., Schekochihin, A. A., and Tatsuno, T.: Kinetic Simulations of Magnetized Turbulence in Astrophysical Plasmas, Phys. Rev. Lett., 100, 065004, doi:10.1103/PhysRevLett.100.065004, 2008.

Helliwell, A.: Whistlers and Related Ionospheric Phenomena. Stanford University Press, Stanford, CA, 1965.

Kraichnan, R. H.: Inertial-Range Spectrum of Hydromagnetic Turbulence, Phys. Fluids, 8, 1385-1387, 1965.

Kingsep, A. S., Chukbar, K. V., and Yan'kov, V. V.: Reviews of Plasma Physics, Consultants Bureau, New York, 16, 243-291, 1990.

Gordeev, A. V., Kingsep, A. S., and Rudakov, L. I.: Electron magnetohydrodynamics, Phys. Reports, 243, 215-315, 1994.

Shukla, P. K.: Modulational instability of whistler-mode signals, Nature, 274, 874-875, 1978.

Kolmogorov, A. N.: The Local Structure of Turbulence in Incompressible Viscous Fluid for Very Large Reynolds' Numbers, Dokl. Akad. Nauk SSSR, 30, 301-305, 1941.

Leamon, R. J., Matthaeus, W. H., Smith, C. W., Wong, H. K.: Contribution of Cyclotron-resonant Damping to Kinetic Dissipation of Interplanetary Turbulence, Astrophys. J., 507, L181, doi:10.1086/311698, 1998.

Matthaeus, W. H., Servidio, S., and Dmitruk, P.: Comment on "Kinetic Simulations of Magnetized Turbulence in Astrophysical Plasma" by Howes, G. G., Dorland, W., Cowley, S. C., Hammett, G. W., Quataert, E., Schekochihin, A. A. and Tatsuno, T., Phys. Rev. Lett., 101, 149501, doi:10.1103/PhysRevLett.100.065004, 2008.

Mason, R. J., Auer, P. L., Sudan, R. N., Oliver, B. E., Seyler, C. E., and Greenly, J. B.: Nonlinear magnetic field transport in opening switch plasmas, Phys. Fluids B., 5, 1115-1127, 1993.

Russell, C. T., Zhang, T. L., Delva, M., Magnes, W., Strangeway, R. J., and Wei, H. Y.: Lightning on Venus inferred from whistlermode waves in the ionosphere, Nature, 450(7170), 661-662, 2007.

Servidio, S., Matthaeus, W. H., and Carbone, V.: Statistical properties of ideal three-dimensional Hall magnetohydrodynamics: The spectral structure of the equilibrium ensemble, Phys. Plasmas., 15, 042314-15, 2008.

Scholer, M. and Burgess, D.: Whistler waves, core ion heating, and 
non-stationarity in oblique collisionless shocks, Phys. Plasmas, 14, 072103-11, 2007.

Stenberg, G., Oscarsson, T., André, M., Vaivads, A., BackrudIvgren, M., Khotyaintsev, Y., Rosenqvist, L., Sahraoui, F., Cornilleau-Wehrlin, N., Fazakerley, A., Lundin, R., and Décréau, P. M. E.: Internal structure and spatial dimensions of whistler wave regions in the magnetopause boundary layer, Ann. Geophys., 25, 2439-2451, 2007, http://www.ann-geophys.net/25/2439/2007/.

Stenzel, R. L.: Whistler wave propagation in a large magnetoplasma, Phys. Fluids, 19(6), 857-864, 1976.

Stenzel, R. L.: Self-ducting of large-amplitude whistler waves, Phy. Rev. Lett., 35(9), 574-577, 1975.

Stenzel, R. L. and Urrutia, J. M.: Force-free electromagnetic pulses in a laboratory plasma, Phy. Rev. Lett., 65(16), 2011-2014, 1990.

Stenzel, R. L., Urrutia, J. M., and Rousculp, C. L.: Pulsed currents carried by whistlers, I - Excitation by magnetic antennas, Phys. Fluids B., 5(2), 325-338, 1993.

Urrutia, J. M., Stenzel, R. L., and Rousculp, C. L.: Pulsed currents carried by whistlers, II. Excitation by biased electrodes, Phys. Plasmas, 1(5), 1432-1438, 1994.

Shaikh, D., Das, A., Kaw, P. K., and Diamond, P.: Whistlerization and anisotropy in two-dimensional electron magnetohydrodynamic turbulence, Phys. Plasmas., 7, 571-579, 2000.

Shaikh, D., Das, A., and Kaw, P. K.: Hydrodynamic regime of twodimensional electron magnetohydrodynamics, Phys. Plasmas, 7, 1366-1373, 2000.
Shaikh, D. and Zank, G. P.: Anisotropic Turbulence in Twodimensional Electron Magnetohydrodynamics, Astrophys. J., 599, 715-722, 2003.

Shaikh, D.: Generation of Coherent Structures in Electron Magnetohydrodynamics, Physica Scripta, 69, 216, doi:10.1238/Physica.Regular.069a00216, 2004.

Shaikh, D.: Theory and Simulations of Whistler Wave Propagation, J. Plasma Phys., 75, 117, doi:10.1017/S0022377808007198, 2008.

Shaikh, D.: Whistler Wave Cascades in Solar Wind Plasma, Mon. Not. Royal Astrono. Soc., in press, 2009.

Shaikh, D. and Zank, G. P.: Driven dissipative whistler wave turbulence, Phys. Plasmas, 12, 122310-7, 2005.

Shaikh, D. and Shukla, P. K.: Three Dimensional Simulations of Compressible Hall MHD Plasmas, AIPC, FRONTIERS IN MODERN PLASMA PHYSICS: 2008 ICTP International Workshop on the Frontiers of Modern Plasma Physics, AIP Conference Proceedings, 1061, 66-75, 2008.

Shaikh, D. and Shukla, P. K.: 3-D simulations of fluctuation spectra in the Hall-MHD plasma, Phys. Rev. Lett., 102, 045004, doi:10.1103/PhysRevLett.102.045004, 2009.

Wei, X. H., Cao, J. B., Zhou, G. C., Santol, O., Rôme, H., Dandouras, I., Cornilleau-Wehrlin, N., Lucek, E., Carr, C. M., and Fazakerley, A.: Cluster observations of waves in the whistler frequency range associated with magnetic reconnection in the Earth's magnetotail, J. Geophys. Res., 112(A10), A10225, doi:10.1029/2006JA011771, 2007.

Zhou, H. B., Popadopolous, K., Sharma A. S., and Chang, C. L.: Electronmagnetohydrodynamic response of a plasma to an external current pulse, Phys. Plasmas., 3, 1484-1494, 1996. 